\title{
Retos para la educación médica en México en los tiempos del COVID-19
}

\section{Challenges for medical education in Mexico in the face of COVID-19}

\author{
Aldo Barajas-Ochoa, ${ }^{1 *}$ Jorge S. Andrade-Romo ${ }^{2}$ y Vicente O. Ramos-Santillán ${ }^{3}$ \\ ${ }^{1}$ Rutgers New Jersey Medical School, Department of Medicine, Nueva Jersey; ${ }^{2}$ New York Eye and Ear Infirmary, Icahn School of Medicine, Mount \\ Sinai, Nueva York; ${ }^{3}$ Montefiore Medical Center, Albert Einstein College of Medicine, Nueva York. Estados Unidos
}

\section{Introducción}

Al 2 de abril de 2020, solo 116 días desde la descripción del primer caso por infección del virus SARS-CoV2, causante de la enfermedad COVID- 19, se han registrado 1014673 casos y 50030 muertes en 181 países. ${ }^{1}$ En Estados Unidos se han registrado 244678 casos y 5911 muertes (tan solo en el estado de Nueva York hay 93053 casos y 2538 muertes), mientras que en México se han registrado 1378 casos y 37 muertes. Se espera que este número siga aumentando en ambos países. El sistema de salud en México, así como el resto del mundo, afrontará una problemática descomunal en los meses venideros.

El sistema de educación médica de México debe adaptarse a los requerimientos asistenciales generados por la pandemia. El internado de pregrado, el servicio social y el sistema nacional de residencias médicas son ciclos esenciales en la formación del médico en México. Los médicos internos de pregrado, pasantes y residentes representan una parte esencial de los médicos que realizan tareas asistenciales y que estarán expuestos a pacientes con COVID-19. Entre 2017, 2018 y 2019, en México 26972 médicos (8480, 8821 y 9671 , respectivamente) fueron aceptados en el Sistema Nacional de Residencias Médicas. ${ }^{2}$ Asimismo, de acuerdo con el anuario estadístico de educación superior 2018-2019 de la Asociación Nacional de Universidades e Instituciones de Educación Superior, ${ }^{3}$ había 139272 estudiantes de la carrera de medicina general, con 16070 graduados de la licenciatura. Asumiendo que el número de graduados es similar al de médicos pasantes del servicio social y de médicos internos de pregrado, hay aproximadamente 32000 médicos pasantes e internos de pregrado en México. La suma de lo anterior da un aproximado de 58972 médicos en la fase clínica del sistema de educación médica. Sin embargo, la carga asistencial y las responsabilidades durante cada etapa son distintas, lo cual exige que se definan los papeles asistenciales y las estrategias de reclutamiento, y se clarifique el nivel de riesgo al que estarán expuestos los médicos en formación.

Los autores de esta carta somos médicos educados en el sistema de educación médica de México y en este momento nos encontramos en el centro de la pandemia causada por el virus SARS-CoV2, el área metropolitana de la ciudad de Nueva York. Hemos experimentado los cambios en el estilo de vida en el área académica y asistencial, y hemos observado las medidas de protección tomadas por las instituciones ante esta emergencia. Creemos que algunas de nuestras observaciones pueden ser de utilidad para el sistema de educación médica en México.

\section{Garantizar la disponibilidad de insumos para protección personal}

Independientemente del nivel de adiestramiento se debe garantizar la disponibilidad de insumos para cumplir con las medidas de protección personal; para asistir a los pacientes con COVID-19 se requiere un cubrebocas regular o máscara quirúrgica (o N95 para procedimientos que generan aerosol, como la intubación
Correspondencia:

*Aldo Barajas-Ochoa

E-mail: aldouch5@gmail.com
Fecha de recepción: 04-04-2020

Fecha de aceptación: 13-04-2020

DOI: 10.24875/GMM.20000161
Gac Med Mex. 2020;156:254-257

Disponible en PubMed

www.gacetamedicademexico.com

0016-3813/@ 2020 Academia Nacional de Medicina de México, A.C. Publicado por Permanyer. Este es un artículo open access bajo la licencia CC BY-NC-ND (http://creativecommons.org/licenses/by-nc-nd/4.0/). 
endotraqueal), protección ocular (gafas o caretas de protección), guantes y batas de aislamiento. El uso correcto del equipo de protección personal disminuye la probabilidad de infección nosocomial, reduce el número de médicos que deben ser hospitalizados 0 puestos cuarentena, y la la probabilidad de transmisión por los mismos a la población. De esta forma, el sistema de salud cuenta con más médicos disponibles para tratar pacientes, a la vez que disminuye el número de individuos infectados. Sin embargo, en etapas tempranas de la emergencia sanitaria ya se ha reportado la falta de insumos y protocolos en lugares como el Instituto Nacional de Enfermedades Respiratorias; ${ }^{4}$ compañeros residentes de todo el país han expresado preocupación por no contar en sus hospitales con los insumos para cumplir con las medidas de protección personal, además de otros recursos. México no es la única nación con esta problemática, la cual también está ocurriendo en Estados Unidos e Italia, ${ }^{5}$ los dos países con más casos registrados de la enfermedad a la fecha, ${ }^{1}$ en los cuales varios hospitales se han visto obligados a depender de donaciones de instituciones externas o de particulares.

\section{Definir el riesgo de exposición aceptable de acuerdo con el nivel de adiestramiento}

Debe definirse el riesgo de exposición aceptable de los médicos en entrenamiento. Aplaudimos la iniciativa tomada por la Dirección General de Calidad y Educación en Salud (DGCES) para proteger al personal en formación. ${ }^{6}$ A partir del 24 de marzo, todos los médicos internos de pregrado no deberían estar presentes en áreas de riesgo para COVID-19. Históricamente, los médicos internos de pregrado tienen la doble condición de estudiante ${ }^{7}$ y trabajadorempleado. ${ }^{8,9}$ Esta medida de la DGCES denota que el bienestar del médico interno de pregrado se sobrepone a sus labores asistenciales y resalta el papel del médico interno de pregrado como estudiante más que como empleado. No obstante, si los servicios de salud son saturados por el número de enfermos durante la pandemia, se podría considerar colocar a los médicos internos de pregrado donde sean necesarios.

La DCGES no suspenderá las actividades asistenciales de los médicos pasantes en servicio social y residentes, además de establecer que estos médicos deben recibir el equipamiento necesario para su protección en la atención de pacientes sospechosos y confirmados de COVID-19. El marco jurídico que define las posiciones de los pasantes en servicio social hace que sea complejo determinar el nivel de obligaciones asistenciales que se les debe asignar durante la pandemia. ${ }^{10,11}$ Sin embargo, la labor de estos médicos es esencial para el sistema de atención en salud del primer nivel, pues cerca de una tercera parte de las unidades de atención primaria del sistema público es cubierta por ellos. ${ }^{12}$ La DGCES establece que los médicos residentes continuarán con sus actividades de práctica clínica según su programa académico.

Adicionalmente, es crucial definir el nivel de exposición de los estudiantes de medicina que se encuentren en rotaciones clínicas, ¿cuál es el papel del estudiante en el ámbito clínico durante esta pandemia? Desafortunadamente, a diferencia de otras situaciones que requieren un incremento de atención médica, como desastres naturales en los que el estudiante puede aprender, en esta pandemia los estudiantes pueden actuar como agentes de transmisión asintomáticos, además de disminuir los insumos de protección personal y las pruebas de COVID-19 disponibles en caso de que enfermen.

Lo anterior llevó a múltiples escuelas de medicina de Estados Unidos a adoptar medidas inmediatas de distanciamiento social y a restringir el acceso de los estudiantes de medicina a las zonas asistenciales, ${ }^{13}$ lo que ha generado nuevos desafíos para la enseñanza médica. Si bien antes de la pandemia algunas asignaturas ya se impartían en línea en las escuelas de medicina de Estados Unidos, las medidas de distanciamiento social requirieron que todos los cursos de ciencias básicas fueran migrados a este formato. El distanciamiento social afectó de forma significativa a la enseñanza en los campos clínicos, al limitar las interacciones paciente-estudiante. Aún se están desarrollando estrategias alternativas, que incluyen la utilización de casos virtuales y el uso de telesalud, entre otras. ${ }^{13}$

De forma oportuna, la DCGES suspendió las actividades académicas en campos clínicos para los estudiantes en áreas de la salud a partir del 23 de marzo. Asimismo, la educación de los estudiantes de medicina que cursan los primeros años de la carrera se verá afectada por la interrupción de las clases presenciales en las instituciones educativas. Los primeros pasos necesarios para mitigar la transmisión de COVID-19 han afectado el paradigma de la educación en salud en México, por lo que será importante evaluar las consecuencias de estas acciones. Es posible que el sistema de educación de pregrado en medicina deba ajustarse a un sistema de educación 
en línea en el futuro inmediato, lo que requerirá del esfuerzo de todos los miembros de la comunidad académica médica para resolver los nuevos retos.

\section{Ajustar las actividades clínicas de residentes de diferentes especialidades de acuerdo con la carga asistencial}

Múltiples sistemas de salud en la ciudad de Nueva York tomaron acciones para evitar la transmisión nosocomial, incluyendo la cancelación de todos los procedimientos quirúrgicos electivos, y numerosos quirófanos han sido transformados en unidades de terapia intensiva para incrementar la disponibilidad de dispositivos de ventilación mecánica. Lo anterior derivó en disminución de la carga asistencial de los médicos residentes de especialidades quirúrgicas, quienes quedaron en "estado de reserva" y se incorporaron a los equipos de respuesta a la pandemia. Ya que en ocasiones los residentes de especialidades quirúrgicas no cuentan con suficiente experiencia en el manejo de pacientes con enfermedades no quirúrgicas complejas, fue esencial la creación de equipos multidisciplinarios en los que personal con mayor experiencia en el manejo de enfermedades médicas funge como líder del grupo. Es posible que esta estrategia sea necesaria en México. Por otro lado, la mayoría de los pacientes con COVID-19 son admitidos a los servicios de medicina interna o terapia intensiva, donde la carga asistencial es abrumadora.

Asimismo, diversos hospitales han creado protocolos o guías de práctica clínica para el manejo de pacientes con COVID-19, los cuales se basan en la evidencia, en los insumos y en el personal disponible en la institución. Estos protocolos son actualizados de forma continua de acuerdo con los nuevos hallazgos sobre la enfermedad. Seguir un tratamiento uniforme y basado en la evidencia permite que el personal de salud en entrenamiento aprenda a manejar la enfermedad del coronavirus 2019. La creación de estos protocolos por los hospitales de México permitirá estandarizar el tratamiento de los pacientes con COVID-19 en cada institución.

\section{Establecer medidas para mantener la salud mental}

El personal asistencial que participe en el equipo de respuesta a la pandemia estará expuesto a altos niveles de estrés. Las fuentes de estrés incluyen presenciar escenas de sufrimiento humano, el riesgo a la salud al que se está expuesto, la toma de decisiones de vida o muerte, las cargas asistenciales intensas, la carencia de recursos y la separación de la familia. Las instituciones para las que trabajamos quienes escribimos esta carta han establecido diferentes estrategias para el manejo de estrés en los trabajadores de la salud; es importante que sean planeadas para los médicos en formación que participen en la pandemia.

\section{Adaptar el reclutamiento de nuevos residentes a las medidas de distanciamiento social}

Finalmente, si bien el reclutamiento de médicos pasantes e internos de pregrado en sus plazas asistenciales podría no estar afectado, este no es el caso con los médicos residentes. El Examen Nacional para Aspirante a Residencias Médicas (ENARM) históricamente ha requerido que los médicos viajen a las sedes de aplicación del examen, lo que genera grandes congregaciones. EI XLIV ENARM se realizará del 25 al 30 de septiembre de $2020,{ }^{14}$ sin embargo, se ha estimado que el distanciamiento social tendrá que estar activo hasta que se creen vacunas o estrategias farmacológicas para tratar la enfermedad, es decir, quizá más de 18 meses..$^{15}$ ¿Cuáles son las medidas que se tomarán para adaptar la selección de los médicos residentes?

\section{Conclusiones}

Sin duda, la sociedad mexicana deberá tomar decisiones difíciles al enfrentar la pandemia generada por el virus SARS-CoV2. El sistema de salud debe estar preparado para brindar cuidados a los pacientes que requieran atención médica al contraer COVID-19. Se requerirá adaptar el sistema de educación médica en México para sortear de la mejor manera los retos generados por la pandemia, así como definir el riesgo de exposición aceptable de acuerdo con el nivel de adiestramiento, garantizar que los insumos para la protección personal estén disponibles, ajustar la carga asistencial de acuerdo con el flujo de pacientes, proteger la salud mental de los médicos en aprendizaje y planear el reclutamiento y la educación de los nuevos médicos que se integrarán al sistema de educación en medicina. Sin duda, esta no es una lista exhaustiva y existirán muchas otras situaciones que deberán ser consideradas en el futuro, pero esperamos que estas reflexiones funcionen como 
catalizador de discusiones futuras para la planeación de la educación médica en México en tiempos del COVID-19.

\section{Responsabilidades éticas}

Protección de personas y animales. Los autores declaran que para esta investigación no se han realizado experimentos en seres humanos ni en animales.

Confidencialidad de los datos. Los autores declaran que en este artículo no aparecen datos de pacientes.

\section{Bibliografía}

1. Johns Hopkins University/CoronavirusResource Center [sitio web]. World Map. COVID-19 Dashboard by the Center for Systems Science and Engineering (CSSE) at Johns Hopkins University (JHU). Estados Unidos: Johns Hopkins University; 2020

2. Comisión Interinstitucional para la Formación de Recursos Humanos para la Salud [sitio web]. Reportes académicos. Médicos inscritos, sustentantes, seleccionados y resultados por escuela o facultad de medicina. México: CIFRHS. [Actualizado 2019 Nov 13].

3. Anuario estadístico de educación superior 2018-2019. México: Asociación Nacional de Universidades e Instituciones de Educación Superior; 2020.
4. Miranda P. Personal del INER protesta por falta de protocolos ante Covid-19. El Universal [en línea]. 2020 Mar 17.

5. Ranney ML, Griffeth V, Jha AK. Critical supply shortages-The need for ventilators and personal protective equipment during the Covid-19 pandemic. N Engl J Med. 2020.

6. Dirección General de Calidad y Educación en Salud [sitio web]. Comunicado. Para personal en formación. México: Dirección General de Calidad y Educación en Salud. [Publicado 2020 Mar 23].

7. Norma Oficial Mexicana NOM-234-SSA1-2003, utilización de campos clínicos para ciclos clínicos e internado de pregrado. México: Diario Oficial de la Federación 2005 Ene 06.

8. Frenk, J. La atención médica, la enseñanza de la medicina y el mercado de trabajo para los médicos: el internado en México. Educ Med Salud. 1984;18:329-343.

9. Ríos-Cortázar V, Gasca-García A, Urbina-García R, Flores-Echavarría R, Lloret-Rivas A. Nuevos modelos educativos en el internado médico de pregrado. La participación de la Universidad. Reencuentro. 2005;42:1-16

10. Mazón-Ramírez JJ. El marco jurídico del servicio social. Gac Med Mex. 2012;148:284-291.

11. Illescas-Martínez I, Luis-Reyes A. Reglamentación del servicio social de medicina en México, a más de 80 años de su implantación. Salud y Administración. 2019;6:27-37.

12. Nigenda G. Servicio social en medicina en México. Una reforma urgente y posible. Gac Med Mex 2013:55:519-527.

13. Rose S. Medical student education in the time of COVID-19. JAMA. 2020.

14. Comisión Interinstitucional para la Formación de Recursos Humanos para la Salud [sitio web]. Convocatoria. XLIV Examen Nacional para Aspirantes a Residencias Médicas 2020. México: CIFRHS; 2020.

15. Imperial College COVID-19 Response Team. Impact of non-pharmaceutical interventions (NPIs) to reduce COVID19 mortality and healthcare demand. Reino Unido: Imperial College London; 2020. 